\title{
Processo de Produção e Desenvolvimento de Objetos de Aprendizagem para o LAPREN
}

\author{
Márcia Cristina Moraes, FACIN/PROGRAD/PUCRS \\ mmoraes@pucrs.br \\ Neda da Silva Gonçalves, FAMAT/PROGRAD/PUCRS \\ nsgoncalves@pucrs.br \\ Marilene Jacinto Müller, FAMAT/PROGRAD/PUCRS \\ mmuller@pucrs.br
}

\begin{abstract}
Resumo. Os problemas evidenciados no ensino fundamental e médio tem trazido para a Universidade alunos ingressantes com deficiências nas áreas de Matemática e Língua Portuguesa. Com o objetivo de oferecer a estes alunos uma oportunidade de recuperar os conceitos básicos foi criado o LAPREN (Laboratório de Aprendizagem), que enfoca seu trabalho no uso de objetos de aprendizagem. Neste artigo iremos apresentar o processo de produção e desenvolvimento de objetos de aprendizagem para o LAPREN.
\end{abstract}

Palavras-chave: Objetos de Aprendizagem, Processo de Produção, Laboratório de Aprendizagem

\section{Production Process and Development of Learning Objects for LAPREN}

Abstract. The problems highlighted in the elementary and secondary education has brought to the University students with deficiencies in the areas of Mathematics and Portuguese. In order to offer to these students an opportunity to recover the basic concepts was created LAPREN (Learning Lab), which focuses its work on the use of learning objects. In this paper we present the process of production and development of learning objects for LAPREN.

\section{Introdução}

Os problemas evidenciados no ensino e na aprendizagem de Matemática, em todos os níveis de escolarização, vem sendo tema de discussão em palestras, seminários e encontros de Educação Matemática e tem provocado, nos professores e pesquisadores da área, um grande estado de preocupação.

Pesquisas realizadas com alunos dos semestres iniciais de cursos superiores da área de Ciências Exatas mostram que as disciplinas matemáticas envolvem algumas dificuldades, relacionadas aos conteúdos escolhidos para as ementas e às habilidades necessárias para a aprendizagem desses conteúdos, tais como abstração, generalização, formulação de hipóteses e deduções. Gomes, Lopes e Nieto (2005), referindo-se ao aluno calouro, comentam: "é certo que uma reforma deveria ser iniciada nos ensinos fundamental e médio, no entanto, esse aluno está chegando ao curso superior e nós, professores universitários, não podemos enviá-los de volta." (p. 7). 
Os resultados das avaliações sob a coordenação do INEP, tais como as provas do Sistema de Avaliação da Educação Básica (SAEB), do Exame Nacional do Ensino Médio (ENEM) e do Programa Internacional de Avaliação de Alunos (PISA), mostram que a situação vem se agravando nos últimos anos.

Dessa forma, face às preocupações com as condições de aprendizagem de Cálculo Diferencial e Integral I, docentes da Faculdade de Matemática têm desenvolvido, desde 2006, um projeto de atendimento às dificuldades dos alunos dessa disciplina. Neste projeto participam monitores, estagiários e bolsistas de iniciação científica do Curso de Matemática que auxiliam os alunos a promover uma aprendizagem mais consciente e autônoma. O objetivo do trabalho é detectar os problemas apresentados por alunos na resolução de questões de disciplinas matemáticas e, após identificá-los, oferecer atendimento individual, ou em pequenos grupos, aos alunos propondo a realização de atividades elaboradas pelo grupo envolvido, bem como a participação em oficinas preparadas para revisar conteúdos básicos ou específicos de Cálculo.

Mais ou menos na mesma época, a Universidade, preocupada com a diversidade das condições de aprendizagem dos alunos ingressantes nos cursos de graduação, iniciou um estudo para a construção de um espaço que pudesse auxiliar estes alunos. Este estudo culminou com a inauguração, em 2009, do LOGOS, um complexo interdisciplinar diferenciado, destinado a processos que envolvem ensinar e aprender, oferecendo a alunos e professores a oportunidade de diálogo com saberes de múltiplas áreas, por meio de atividades e recursos diversificados. Dentro do complexo LOGOS está situado o Laboratório de Aprendizagem (LAPREN), que tem como objetivo oportunizar aos alunos apoio pedagógico, individual e/ou coletivo, na construção de conceitos básicos por meio de atividades de aprendizagem. As atividades de aprendizagem do LAPREN estão centradas no uso de objetos de aprendizagem para as áreas de Matemática e Língua Portuguesa.

Neste sentido, desde a inauguração do LAPREN, em novembro de 2009, o atendimento aos alunos das disciplinas de Cálculo Diferencial e Integral I vem sendo realizado no LAPREN. Para dar suporte a este atendimento, e proporcionar aos alunos materiais interativos que estimulem o entendimento dos conceitos matemáticos, estão sendo desenvolvidos objetos de aprendizagem. No presente artigo, iremos descrever o processo de elaboração e produção destes objetos de aprendizagem e apresentaremos um exemplo de um objeto desenvolvido para a área de Matemática.

\section{Referencial Teórico}

Nesta seção iremos apresentar o Laboratório de Aprendizagem (LAPREN) e o trabalho que vem sendo realizado pela Faculdade de Matemática neste laboratório. Também descreveremos os conceitos de objetos de aprendizagem que estão servindo de base para o desenvolvimento do trabalho.

\subsection{Laboratório de Aprendizagem - LAPREN}

O Laboratório de Aprendizagem (LAPREN) foi inaugurado em novembro de 2009 pela Pró-Reitoria de Graduação da Universidade com o objetivo oportunizar, aos alunos da Graduação, apoio pedagógico a situações específicas de aprendizagem, de modo a auxiliá-los no desenvolvimento de competências básicas. Entendemos que este desenvolvimento se baseia em uma perspectiva interacionista e relacional (Becker, 
2001), através de uma mediação pedagógica que envolve a relação aluno-monitorconhecimento, dando ênfase no protagonismo, autoria e autonomia do aluno.

O público-alvo do laboratório são os alunos de Graduação e Pós-graduação dos diferentes cursos e programas da Universidade, que procuram o laboratório por iniciativa própria ou por recomendação dos professores, com vistas a ampliar suas condições de aprendizagem significativa. Os alunos são recebidos no laboratório por monitores. Os monitores são alunos de iniciação científica da Universidade, das áreas de Língua Portuguesa e Matemática, que realizam atividades relacionadas ao atendimento aos alunos e atividades de pesquisa. Dentro das atividades de atendimento ao aluno, os monitores:

- recebem os alunos, diagnosticam, com o auxílio de um professor, as necessidades destes alunos e os informam sobre os materiais disponíveis no laboratório que estão relacionados as necessidades identificadas;

- registram a presença do aluno, bem como os conteúdos e os procedimentos que estão sendo utilizados, para garantir a continuidade do trabalho;

- fazem a mediação da aprendizagem, se necessário, auxiliando a interação do aluno com os materiais específicos.

Dentro das atividades de pesquisa, os monitores:

- avaliam criticamente os objetos de aprendizagem elaborados, sugerindo outros com base nas necessidades identificadas nos alunos;

- participam da elaboração de objetos de aprendizagem identificados pela sua própria necessidade como ex-alunos das disciplinas relacionadas ao laboratório.

O aluno, por sua vez, pode:

- utilizar as dependências do LAPREN para realizar estudos, com ou sem a utilização de recursos específicos do laboratório e/ou sem a orientação dos monitores;

- organizar seu estudo por módulos de ensino individualizado, sob orientação dos monitores, a partir de uma proposta de atendimento específico às suas necessidades e interesses;

- decidir sobre a extensão e/ou aprofundamento do estudo.

A Faculdade de Matemática, com a utilização deste espaço, e em continuidade as demais atividades já desenvolvidas nos anos anteriores, vinculadas a monitoria de disciplina, pretende dar prosseguimento ao trabalho de monitoria, buscando aprimorar, cada vez mais, o auxílio aos alunos nas disciplinas matemáticas, principalmente de primeiro semestre. O fato dos monitores serem bolsistas de Iniciação Científica, alunos do Curso de Matemática, vem ao encontro da proposta dos professores do Curso de Licenciatura Plena em Matemática da Universidade de desenvolver um currículo que efetivamente incorpore uma profunda relação entre teoria e prática e que possibilite ao aluno a realização de trabalhos de investigação e de pesquisa. É de extrema importância que se consolide a pesquisa como uma atividade inerente ao sistema educativo e à formação inicial e continuada de professores, sendo essa compreendida como prática solidária que envolva licenciandos e seus professores, bem como alunos de diversos cursos da Universidade. Encontramos em Ponte, Brocardo e Oliveira (2003) que "Em experiências já empreendidas com trabalho investigativo, os alunos têm mostrado realizar aprendizagens de grande alcance e desenvolver um grande entusiasmo pela Matemática."(p.10). É evidente que investigar é uma excelente forma de construir 
conhecimento e, atualmente observa-se, em especial no ensino de Matemática, ser esta uma maneira de motivar os alunos para a aprendizagem.

\subsection{Objetos de Aprendizagem}

Atualmente, não existe uma definição única e de consenso de todos os pesquisadores da área, do que sejam objetos de aprendizagem. De acordo com Downes (2004), existem várias definições, sendo algumas consideradas muito amplas e outras muito específicas. Sosteric e Hesemeir (2004) consideram que isto ocorre devido ao diferente entendimento que os pesquisadores de diferentes áreas fazem do conceito de objetos de aprendizagem.

De acordo com a IEEE (2008), objetos de aprendizagem, ou OA, podem ser entendidos como "qualquer entidade digital ou não digital que possa ser usada, reusada ou referenciada durante aprendizagem suportada pela tecnologia". Este é um exemplo de definição bastante ampla, pois qualquer material instrucional pode ser considerado um objeto de aprendizagem.

Para Sosteric e Hesemeier (2004), objetos de aprendizagem são arquivos digitais (imagens, filmes, etc) que tem a intenção de serem usados para propósitos pedagógicos e que incluem, internamente ou por associação, sugestões sobre o contexto apropriado para uso do objeto. De acordo com os autores, esta definição limita o universo dos objetos de aprendizagem, seguindo o que é apontado pela literatura e prática atual da área.

Os objetos de aprendizagem produzidos, por diferentes instituições e pesquisadores, são catalogados em repositórios. Um repositório é um local, normalmente integrado a um sistema de aprendizagem, no qual ficam organizados e armazenados os OA com o objetivo de acessar o OA desejado com maior precisão e facilidade, sendo o acesso para utilização ou para fins de reuso em outras aplicações (Downes, 2004).

Um exemplo de repositório é o DSpace. O DSpace é um repositório digital desenvolvido pelas bibliotecas do MIT (Massachusetts Institute of Technology) em conjunto com a HP (Hewlett-Packard), que tem como objetivo armazenar, preservar, gerir e disseminar o produto intelectual dos seus pesquisadores. O DSpace é disponibilizado livremente às instituições de pesquisa, sob a forma de um produto de código aberto, que pode ser livremente adaptado e expandido funcionalmente (Smith et al. 2003) (Duraspace, 2010).

\section{Processo de Produção e Desenvolvimento dos Objetos de Aprendizagem}

$\mathrm{O}$ processo de produção e desenvolvimento dos objetos de aprendizagem teve inicio com a formação da equipe interdisciplinar que iria fazer parte do projeto. A equipe foi composta por oito professores: três da Faculdade de Letras, dois da Faculdade de Matemática, um da Faculdade de Informática e dois da Faculdade de Educação. Além dos professores, compunha a equipe um funcionário responsável pelas questões de layout e comunicação, dois funcionários da gerência de tecnologia e quatro monitores de programação.

As primeiras reuniões da equipe foram destinadas a elaboração do layout a ser utilizado nos objetos de aprendizagem, considerando os aspectos de identidade visual da universidade. Nestas reuniões foram definidos os padrões de interface, tipo e tamanho de fonte, uso de links, botões e boas práticas para uso de textos na Web. Foi definido que os objetos de cada área irão ser identificados através de cores. As cores definidas até 
o momento foram tons de marrom e amarelo para a área de Letras e tons de azul para a área de Matemática. Além dos padrões de interface, a equipe definiu que cada objeto irá possuir uma página de apresentação, como mostrado na figura 1 . O canto esquerdo da página de apresentação contém o logo do LOGOS, a identificação da área e subárea do objeto de aprendizagem e o logo da instituição. O restante de página contém o título do objeto de aprendizagem, objetivo, descrição, equipe e referências. Além destas informações, a página contém um link para o "Início" e outro para o "Próximo". Estando na página de apresentação, o link "Início" leva para o repositório institucional e o "Próximo" leva para página de conteúdo do objeto de aprendizagem. O repositório utilizado pela instituição é o DSpace. A equipe optou pela construção da página de apresentação, devido à definição de objetos de aprendizagem dos autores Sosteric e Hesemeier (2004), que indicam que o objeto de aprendizagem deve possuir sugestões sobre o contexto apropriado para o seu uso.

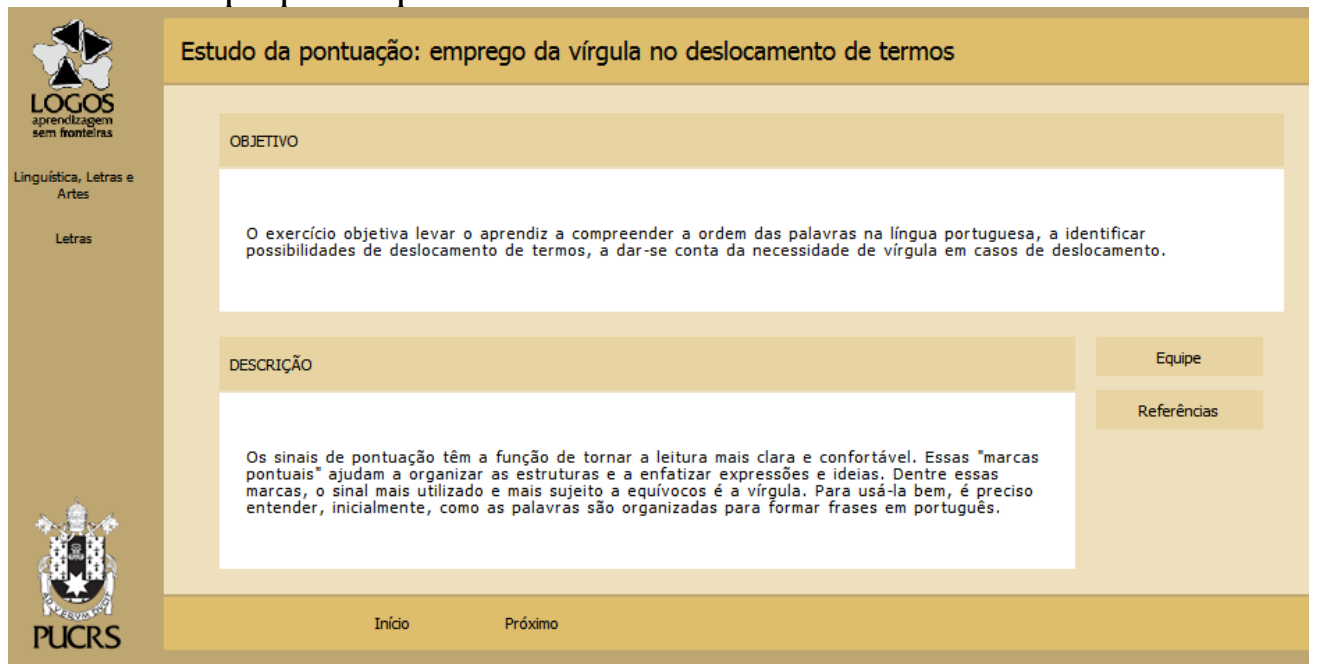

Figura 1: Exemplo de página de apresentação para um objeto da subárea de Letras

Para que a página de apresentação seja elaborada de forma dinâmica, permitindo a rápida construção e atualização da mesma, foi desenvolvido, pela equipe de gerência de tecnologia, o sistema LAPREN. Este sistema foi desenvolvido na linguagem de programação Java e utiliza como banco de dados o Oracle. Além de possibilitar a construção e atualização da página de apresentação do objeto, o sistema faz o upload dos arquivos que compõem o objeto para o servidor, construindo também a navegação entre os arquivos que fazem parte do objeto.

Após o objeto ser desenvolvido pela equipe de professores e implementado pela equipe de programadores, o objeto é colocado no sistema LAPREN para a construção da sua página de apresentação e da navegação entre seus arquivos. Feito isto, o objeto é catalogado no repositório DSpace institucional e está disponível para uso dos alunos.

O processo de desenvolvimento do objeto de aprendizagem está atualmente organizado em quatro atividades fundamentais, como indicado em Sommerville (2003): especificação (define as funcionalidades do software e as restrições em sua operação); desenvolvimento (Projeto e Implementação - produz o software de modo que cumpra sua especificação); validação (valida o software para garantir que ele faça o que foi especificado) e evolução do software (o software precisa evoluir para atender às necessidades mutáveis do cliente). Estas atividades seguem uma abordagem de desenvolvimento incremental (Sommerville, 2003), onde os clientes, no caso os professores, identificam, em um esboço, as funções a serem fornecidas pelo objeto de 
aprendizagem. Eles identificam quais funções são mais importantes e quais são menos importantes para eles. Logo após, é definida uma série de estágios de entrega, com cada estágio fornecendo um subconjunto das funcionalidades do objeto. Uma vez que um estágio é concluído e entregue, os professores podem experimentar o objeto de aprendizagem e esclarecer as funcionalidades para os próximos estágios de entrega.

\subsection{Especificação}

A fase de especificação envolve os professores que irão produzir os objetos de aprendizagem e a equipe que irá realizar o desenvolvimento dos objetos de aprendizagem.

Em um primeiro momento, os professores definem as especificações do objeto de aprendizagem em termos de conteúdo (incluindo a escolha do tema, a forma como será apresentado pedagogicamente e os recursos que serão utilizados). Após, se realiza a fase do roteiro de construção propriamente dito, a qual contém a atividade a ser desenvolvida. Uma das preocupações do grupo é a de que cada objeto construído seja claro e simples sem muita sofisticação de apresentação. Os recursos tecnológicos que serão usados devem ter como objetivo apenas o êxito da aprendizagem. Neste momento, os professores elaboram um storyboard inicial com o roteiro previsto para o objeto de aprendizagem.

A fase de elaboração do storyboard demanda tempo dos professores, pois considerando que o objetivo principal é à busca de autonomia dos alunos em sua aprendizagem, cada objeto deve levar a uma real noção do conceito trabalhado, apresentar características que despertem atenção por sua consistência e ao mesmo tempo incentivar o seu uso por causar o prazer de proporcionar algo que vai ampliar o conhecimento. O storyboard pode ser elaborado usando o PowerPoint ou o Word, por exemplo. Além deste storyboard, os professores preenchem a ficha de cadastro do objeto de aprendizagem. Os dados constantes nesta ficha serão utilizados na fase de desenvolvimento e para implantação do objeto de aprendizagem no repositório DSpace institucional.

Após ter realizado o storyboard, os professores se reúnem com a equipe de desenvolvimento para explicar o funcionamento do objeto. Neste momento, a equipe de desenvolvimento sugere aos professores possibilidades de implementação do objeto em relação às interações entre as telas, animações e exercícios. O objetivo desta reunião é que a equipe de desenvolvimento compreenda o que deve ser desenvolvido e indique possibilidades de implementação para o objeto de aprendizagem.

\subsection{Desenvolvimento (Projeto e Implementação)}

Nesta fase, a equipe de desenvolvimento faz o mapeamento do storyboard construído pelo professor para o objeto de aprendizagem. Os objetos de aprendizagem são desenvolvidos em Flash e este desenvolvimento consiste nas seguintes etapas:

- A parte gráfica (fundo, botões e imagens) é desenvolvida no Fire Works.

- Os textos e parte gráfica (gerados no Fire Works) são colocados no Flash.

- São adicionados os efeitos e animações no Flash.

- Os conteúdos do objeto são formatados em Flash.

Após os conteúdos do objeto estarem formatados em Flash, o objeto é cadastrado no sistema LAPREN, para que seja construída dinamicamente a página de apresentação deste objeto. Os arquivos que compõem o objeto são enviados para o 
servidor através do sistema LAPREN, a fim de que o objeto possa futuramente ser acessado pelo repositório DSpace institucional.

Durante a fase de desenvolvimento, os desenvolvedores utilizam a prática de reuso de código, buscando em outros objetos padrões e códigos que possam ser reaproveitados.

\subsection{Validação}

A fase de validação é composta pelos testes de unidade, módulo, sistema e aceitação (Sommerville, 2003). Os três primeiros testes são realizados pela equipe de desenvolvimento e o último teste é realizado tanto pela equipe de desenvolvimento quanto pelos professores.

No teste de unidade são testados os componentes individuais do objeto de aprendizagem, como imagens, tabelas, botões e animações. No teste de módulo é testada a integração das unidades independentes com a seqüência de conteúdos do objeto de aprendizagem. No teste de sistema, o objeto é colocado no sistema LAPREN e são testadas as navegações entre as páginas do objeto e sua página de apresentação. No teste de aceitação a equipe de desenvolvimento e os professores realizam uma revisão conjunta, que pode levar a alterações tanto de conteúdo quando de programação. Somente após ter passado com sucesso pelo teste de aceitação é que o objeto de aprendizagem é cadastrado no sistema DSpace institucional e fica disponível para os alunos.

\subsection{Evolução do software}

Após a colocação do objeto no repositório, caberá aos monitores analisar o objeto considerando seus próprios julgamentos e também os dados obtidos quando da observação do uso do objeto pelos alunos. Esta análise poderá direcionar aperfeiçoamentos a serem realizados no objeto de aprendizagem. Desta maneira, o objeto pode sofrer manutenções quando necessário.

$\mathrm{Na}$ próxima seção iremos apresentar brevemente um objeto que faz parte do respositório DSpace institucional, o objeto "Problemas de maximação e minimação (uma variável)".

\section{Exemplo de Objeto de Aprendizagem da Área de Matemática}

O objeto "Problemas de maximação e minimação (uma variável)" foi criado para auxiliar os alunos no conteúdo sobre Aplicações de Derivadas, mais especificamente no que se refere à resolução de problemas. Em qualquer conteúdo da disciplina de Cálculo Diferencial e Integral, a resolução de problemas tem-se apresentado como um dos maiores obstáculos para a aprendizagem.

Depois de alguma reflexão e discussão sobre o objetivo do trabalho e a forma como esperávamos que atingisse os alunos, decidimos que após sugerir uma leitura sobre resolução de problemas, passaríamos a trabalhar diretamente com alguns problemas escolhidos nas bibliografias da disciplina, sempre chamando atenção e retomando os conteúdos que apareciam em cada situação.

Ao realizar uma pesquisa no repositório institucional através de palavras-chave, título ou assunto, o aluno acessa o objeto, encontrando a página de apresentação do mesmo, como mostra a figura 3. 
Ao entrar no objeto propriamente dito e passar pelo seu início (figura 4), muitas vezes o aluno encontrará pequenos textos que comentará o tipo de trabalho a ser feito com os procedimentos aconselhados. Nesse objeto, por exemplo, há links que levarão ao artigo recomendado, a explicações sobre conteúdos que se apresentam como prérequisitos, aos conceitos principais abordados no trabalho, como mostrado nas figuras 5 e 6. Além destes links, o objeto possui textos explicativos e uma bibliografia indicada. Após acessar estes materiais o aluno tem uma série de exercícios para serem realizados.

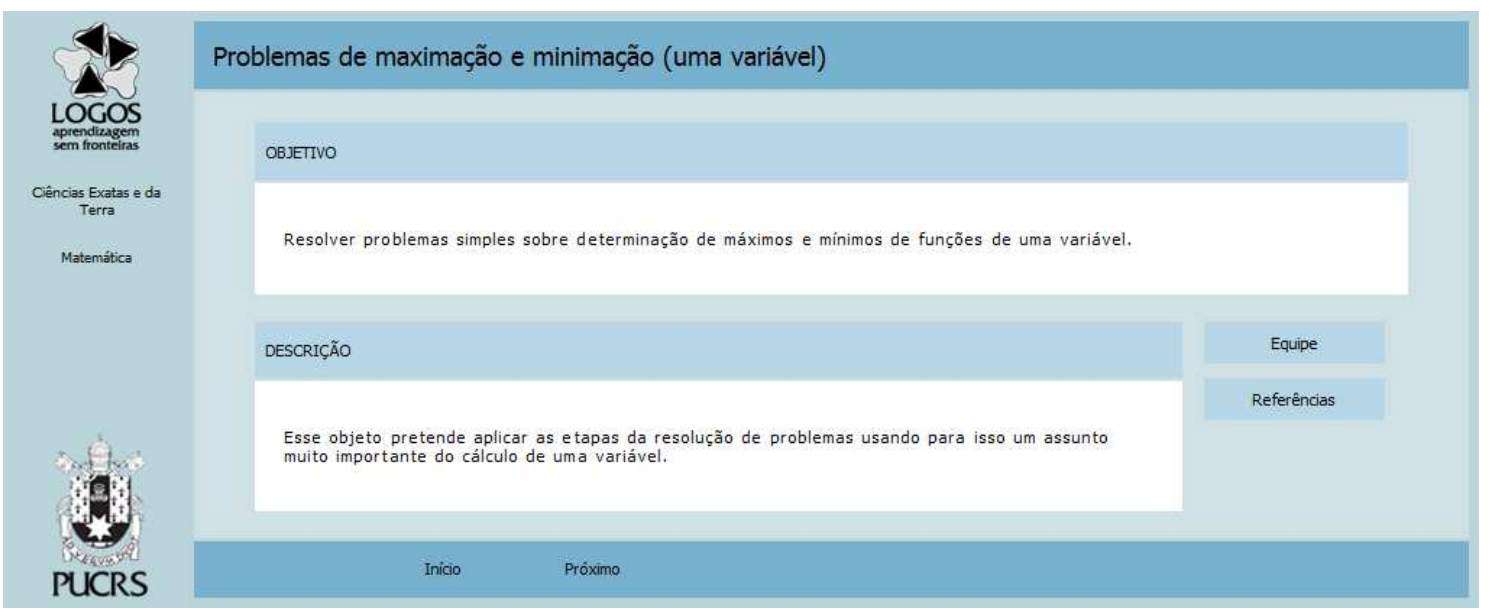

Figura 3: Página de apresentação do objeto Problemas de maximação e minimação

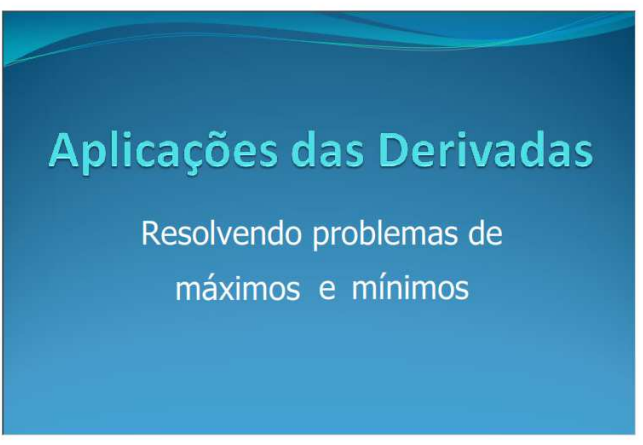

Figura 4: Início do objeto

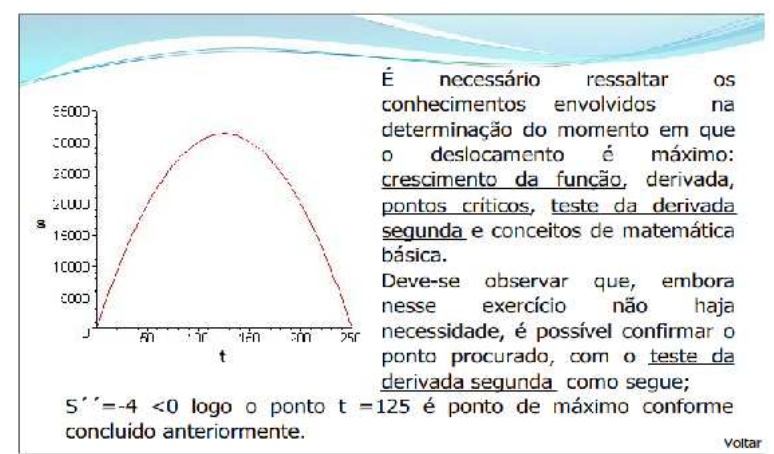

Figura 6: Página com links para reconhecimento dos conceitos envolvidos

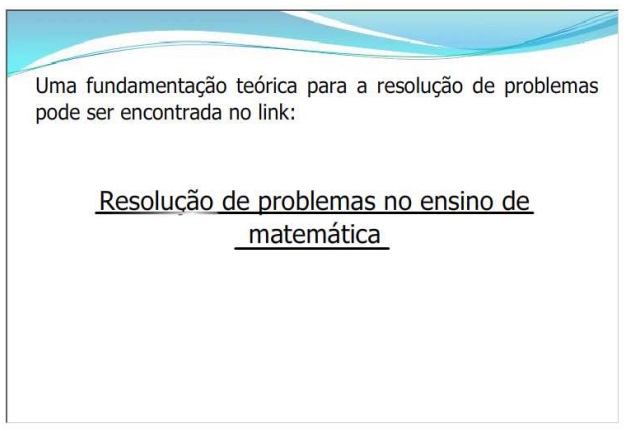

Figura 5: Página de Motivação

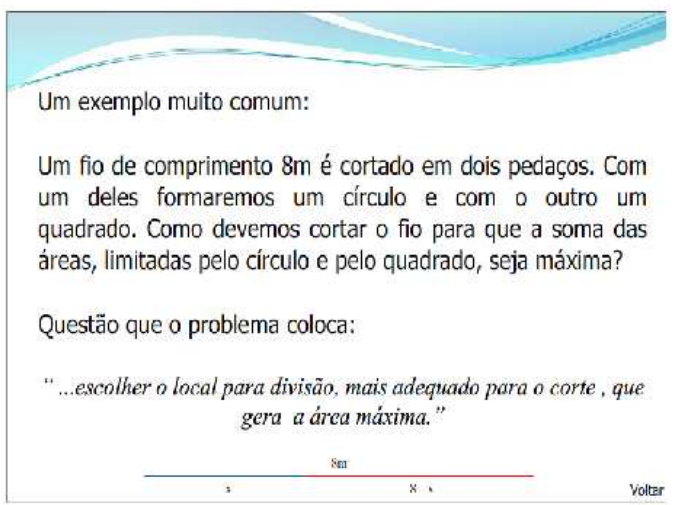

Figura 7: Um problema típico 
Pretende-se com esse objeto, apresentar os conceitos de máximos e mínimos com o uso de problemas simples (figura 7) que surgem quando da introdução do assunto "Aplicações de Derivadas". O objeto coloca um assunto importante de forma mais leve, levando o aluno a encarar esses problemas como outros tantos que fazem parte de sua rotina. Em geral o conteúdo teórico é estudado antes da chamada prática o que pode distanciar a situação problema dos fundamentos necessários para sua resolução. A tentativa é de diminuir essa distância através dos links que são apresentados e da linguagem mais informal.

O objeto leva o aluno a pensar de uma maneira objetiva (figura 8), considerando as suas necessidades de aprendizagem e fazendo com que o aluno reconstrua seus conceitos de forma autônoma.

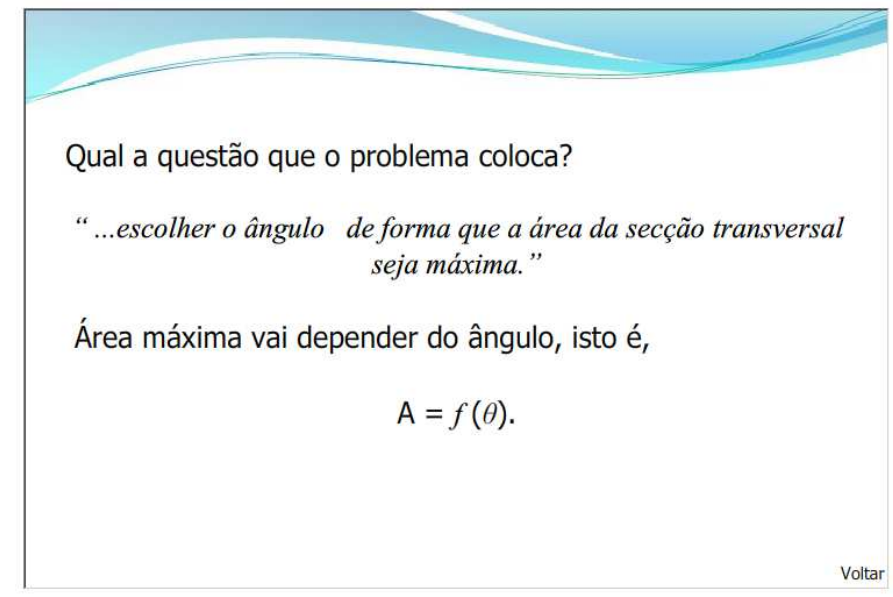

Figura 8: Objetividade na interpretação da situação

\section{Considerações Finais}

A cada início de semestre, temos observado a chegada de calouros na Universidade com problemas conceituais básicos nas áreas de Matemática e Língua Portuguesa. Muitas vezes estes alunos reprovam nas disciplinas iniciais do curso, especialmente, as disciplinas de Cálculo.

Com o objetivo de oferecer aos alunos uma oportunidade de recuperar os conceitos básicos que não foram adequadamente aprendidos no ensino fundamental e médio, foi criado o LAPREN (Laboratório de Aprendizagem). Os alunos que vem ao LAPREN podem realizar seus estudos utilizando objetos de aprendizagem especialmente desenvolvidos para suprir as necessidades, de Matemática e Português, observadas pelos professores das disciplinas iniciais dos cursos.

Neste artigo descrevemos como ocorre o processo de produção e desenvolvimentos dos objetos de aprendizagem para o LAPREN. Desde a inauguração em novembro de 2009 até o momento, produzimos dezenove (19) objetos para a área de Matemática e quatorze (14) objetos para a área de Língua Portuguesa.

Ao final deste semestre (2010/1) realizamos uma pesquisa qualitativa com os alunos freqüentadores do laboratório. Os alunos responderam a um questionário que tinha como objetivo verificar a percepção que o aluno usuário do LAPREN possui em relação à possível contribuição dos objetos de aprendizagem para a sua aprendizagem. Os alunos demonstraram, a partir de suas respostas, que os objetos tem contribuído para 
a sua aprendizagem, como relatado por um aluno de Cálculo I, "Basta dizer que antes não conseguia entender a maioria dos tópicos das aulas, acertava 2 ou 3 exercícios e mais 1 ou 2 pela metade, pensava que era a idade e talvez fosse melhor desistir. Agora, acerto 7 ou 8 dos exercícios e ainda consigo ajudar outros colegas."

A nossa expectativa, que tem sido solidificada, mesmo neste espaço de tempo em que o laboratório está em funcionamento, é de que a maneira com que os assuntos são abordados nos objetos de aprendizagem, apresentando os conceitos de forma diferente dos livros tradicionais e aproximando-os da prática e dia a dia dos alunos, motivem os alunos e levem a uma aprendizagem significativa.

\section{Referências}

BECKER, F. (2001). Educação e Construção do Conhecimento, Artmed Editora.

DOWNES, S. (2004). Learning objects: resources for learning worldwide. In: ONLINE EDUCATION USING LEARNING OBJECTS, R. McGreal, London, Routledge, p. 21-31.

DURASPACE. (2010) "DSpace Manual Release 1.6.2”. Disponível em: <http://www.dspace.org/ 1_6_2Documentation/ DSpace-Manual.pdf>. Acessado em: 30 set. 2010.

GOMES, G. H.; LOPES, C. M. C. e NIETO, S. S. (2005). Cálculo zero: uma experiência pedagógica com calouros nos cursos de engenharia. In: CONGRESSO BRASILEIRO DE ENSINO DE ENGENHARIA, 33, 2005, Campina Grande. Anais...Campina Grande: UFPB, 2005. CD-ROM.

IEEE. (2008). "IEEE Learning Object Metadata". Disponível em: <http://ltsc.ieee.org/wg12/, Agosto>. Acessado em: 30 set. 2010.

PONTE, J, P; BROCARDO, J. e OLIVEIRA, H. (2003). Investigações matemáticas na sala de aula. Belo Horizonte: Autêntica.

SMITH, M. et al. (2003). Dspace: an open source dynamic digital repository. D-Lib Magazine, Volume 9, Número 1. Disponível em: <http://www.dlib.org/dlib/january03/ smith/01smith.html>. Acessado em: 30 set. 2010.

SOSTERIC, M. e HESEMEIER, S. (2004). A first step towards to a theory of learning objects. In: ONLINE EDUCATION USING LEARNING OBJECTS, R. McGreal, London, Routledge, p. 32-42.

SOMMERVILLE, I. Engenharia de software. São Paulo: Pearson Education, 2003. 